\title{
Hydrogen Sulfide Protects Cardiomyocytes against Apoptosis in Ischemia/Reperfusion through MiR-1-Regulated Histone Deacetylase 4 Pathway
}

\author{
Bo Kang ${ }^{a, b}$ Wei Lia Wang Xia Yinghong Yib Yundan Ciren ${ }^{b}$ Hua Shen ${ }^{a}$ \\ Yufeng Zhang ${ }^{\mathrm{a}}$ Huan Jiang $^{\mathrm{a}}{\text { Jian } \mathrm{Xiao}^{\mathrm{a}} \text { Zhinong Wang }}^{\mathrm{a}}$ \\ aDepartment of Cardiothoracic Surgery, Shanghai Changzheng Hospital, Second Military Medical \\ University, Shanghai, bDepartment of Cardiothoracic Surgery, General Hospital of Tibetan Military \\ Command, Lhasa, China
}

\section{Key Words}

Hydrogen sulfide $・$ MicroRNA-1 $・ H D A C 4 \cdot$ Apoptosis $・$ Ischemia-reperfusion injury

\begin{abstract}
Background/Aims: Hydrogen sulfide $\left(\mathrm{H}_{2} \mathrm{~S}\right)$ is a powerful inhibitor of cardiomyocytes apoptosis following ischemia/reperfusion (IR) injury, but the underlying mechanism remains unclear. Our previous study showed that microRNA-1 (miR-1) was upregulated by 2.21 fold in the IR group compared with that in the $\mathrm{H}_{2} \mathrm{~S}$ preconditioned group. MiR-206 affected the process of cardiomyocytes hypertrophy by regulating histone deacetylase 4 (HDAC4). HDAC4 is also known to play an anti-apoptotic role in tumor cells, but its role in the myocardium has not been reported. The aim of this study was to test whether $\mathrm{H}_{2} \mathrm{~S}$ could inhibit apoptosis of cardiomyocytes through HDAC4 regulation by miR-1 in IR. Methods: Cardiomyocytes of neonatal rats were subjected to hypoxia/reoxygenation (HR) injury with or without $\mathrm{H}_{2} \mathrm{~S}$ pretreatment to simulate IR injury. Cardiomyocytes were transfected with miR-1 mimic or HDAC4 siRNA to evaluate whether the miR-1-HDAC4 signaling pathway was involved in the protective effect of $\mathrm{H}_{2} \mathrm{~S}$. Results: HR increased cell apoptosis and caspase- 3 cleavage, upregulated miR-1, and downregulated HDAC4. $\mathrm{H}_{2} \mathrm{~S}$ preconditioning attenuated the apoptosis of cardiomyocytes, caspase- 3 cleavage and LDH release, and enhanced cell viability. In addition, $\mathrm{H}_{2} \mathrm{~S}$ downregulated miR-1, and preserved HDAC4 expression. HDAC4 protein was downregulated by miR-1 mimic. Transfection of cardiomyocytes with miR-1 mimic partially reduced the protective effect of $\mathrm{H}_{2} \mathrm{~S}$. Meanwhile, transfection of cardiomyocytes with siRNA to HDAC4 partially abrogated the protective effect of $\mathrm{H}_{2} \mathrm{~S}$. Conclusions: The miR-1-HDAC4 signaling pathway is involved in the protective effect of $\mathrm{H}_{2} \mathrm{~S}$ against the apoptosis of cardiomyocytes during the IR injury process.




\section{Cellular Physiology Cell Physiol Biochem 2017;41:10-21 \begin{tabular}{ll|l} 
and BiOChemistry & $\begin{array}{l}\text { DOI: 10.1159/000455816 } \\
\text { Published onIIne: January 17, } 2017\end{array}$ & $\begin{array}{l}\text { C } 2017 \text { The Author(s). Published by S. Karger AG, Basel } \\
\text { www.karger.com/cpb }\end{array}$
\end{tabular} \\ Kang et al.: $\mathrm{H}_{2} \mathrm{~S}$ Protects Cardiomyocytes through MiR-1-Regulated HDAC4}

\section{Introduction}

Hydrogen sulfide has been regarded along with carbon monoxide (CO) and nitric oxide (NO) as an endogenous gaseous mediator. Many studies have reported that $\mathrm{H}_{2} \mathrm{~S}$ play an important endogenous protective effect against myocardial IR [1]. However, there are still lots of unanswered questions surrounding its physiological and pathophysiological relevance and roles, notably relating to specific molecular targets of $\mathrm{H}_{2} \mathrm{~S}$ and mechanisms of action.

MicroRNAs (miRNAs) are a recently discovered class of small noncoding RNAs that regulate gene expression. These small noncoding transcripts of 18 to 25 nucleotides modulate protein expression by binding to complementary or partially complementary target mRNAs and thereby targeting the mRNA for degradation or translational inhibition. The discovery of microRNAs has broadened our understanding of the mechanisms that regulate gene expression with the addition of an entirely novel level of regulatory control. Recent studies have demonstrated that microRNA (miR) is involved in multiple physiological and pathological processes including apoptosis by regulating the expression of related genes at the post transcriptional level [2]. MiR-1 is highly expressed and tissue specific in cardiac and skeletal muscles [3]. Our previous study [4] showed that miR-1 was upregulated by 2.21 fold in IR group compared with that in $\mathrm{H}_{2} \mathrm{~S}$ preconditioned group, and that $\mathrm{H}_{2} \mathrm{~S}$ played an unambiguous protective role in cardiomyocytes induced by IR via regulating the expression of miR-1 and apoptosis-related genes.

HDACs, chief mediators of epigenetic gene silencing, remove acetyl groups at the e-amino groups of lysine residues of histones. HDACs are grouped into distinct subfamilies by sequence similarities and structural features. HDAC4 is the member of the class IIa subfamily, which was involved in many physiological and pathological processes. For example, HDAC4 knockout mice display premature ossification of developing bones as a result of early onset of chondrocyte hypertrophy, an effect mediated by loss of HDAC4-induced repression of Runx2. HDAC4 also regulates skeletal muscle differentiation through its interaction with the myogenic transcription factor, MEF2. However, the anti-apoptotic effect of HDAC4 in previous studies was mainly described in cancer cells or skeletal muscle and its role in cardiomyocytes is unresolved. Meanwhile, other studies [5] demonstrated that miR-206 affected the process of cardiomyocyte hypertrophy by regulating histone deacetylase 4 (HDAC4). It suggests that some miRs may regulate the expression of HDAC4. So, the purpose of the present study was to evaluate whether the miR-1 regulated HDAC4 pathway was involved in the protective effect of $\mathrm{H}_{2} \mathrm{~S}$ on IR-induced apoptosis in cariomyocytes.

\section{Materials and Methods}

Isolation of neonatal cardiomyocytes and cell culture

Cardiac ventricle myocytes were isolated from 3-day-old new born Sprague-Dawley (SD) rats, and cultured as described previously [6].

\section{Establishment of the in vitro cardiomyocyte hypoxia/reoxygenation (HR) model}

To simulate myocardial IR in vivo, cardiomyocytes were cultured in serum-free DMEM for $24 \mathrm{~h}$ in a $37^{\circ} \mathrm{C}$ incubator with a hypoxic atmosphere (3\% 02, 92\% N2, and 5\% CO2), and then in a reoxygenation atmosphere (5\% CO2, and $95 \%$ air) at $37^{\circ} \mathrm{C}$ for 2 has described previously [4].

To determine the role of $\mathrm{H} 2 \mathrm{~S}$ in cardiomyocyte apoptosis induced by HR, 10, 30, 50 or $100 \mu \mathrm{M}$ NaHS (an $\mathrm{H} 2 \mathrm{~S}$ donor) was added to the medium at $30 \mathrm{~min}$ prior to HR as described previously [7]. The cardiomyocytes were assigned to six groups: control group (CON group), in which the cardiomyocytes were cultured for $26 \mathrm{~h}$ under normal oxygenation conditions ( $5 \% \mathrm{CO} 2$, and 95\% air); HR group, in which the cardiomyocytes were subjected to HR; and four (S10+HR, S30+HR, S50+HR, S100+HR) H2S precondition groups, in which the cardiomyocytes were pretreated with $10,30,50$, or $100 \mu \mathrm{M}$ NaHS and then subjected to HR. 


\section{Cellular Physiology Cell Physiol Biochem 2017;41:10-21 \begin{tabular}{l|l} 
and Biochemistry Publishe.1159/000455816 & $\begin{array}{l}\text { (c) } 2017 \text { The Author(s). Published by S. Karger AG, Basel } \\
\text { www.karger.com/cpb }\end{array}$
\end{tabular}}

Kang et al.: $\mathrm{H}_{2} \mathrm{~S}$ Protects Cardiomyocytes through MiR-1-Regulated HDAC4

Establishment of the in vivo myocardium IR injury model

SD rats $(250-300 \mathrm{~g})$ were anesthetized with $10 \%$ chloral hydrate $(300 \mathrm{mg} / \mathrm{kg})$ intraperitoneally (i.p.) before endotracheal intubation. IR was induced by ligating the left anterior descending artery for $30 \mathrm{~min}$, followed by loosening the ligature for $120 \mathrm{~min}$, as described previously [4].

Twenty-four rats were equally randomized to three groups: Sham group, in which the rats underwent thoracotomy without ligation; IR group, in which the rats were treated with IR; and H2S group, in which the rats were administered $30 \mu \mathrm{mol} / \mathrm{kg}$ NaHS i.p. for $30 \mathrm{~min}$ prior to IR in addition to the same treatment as that in IR group, as described previously [4].

Lactate dehydrogenase (LDH) assay

The conditioned cell culture supernatant $(200 \mu \mathrm{l})$ was collected after HR to determine LDH levels using a spectrophotometric kit (Roche Diagnostics), according to the manufacturer's instructions, as previously described [8].

MTT assay

After HR injury, cell viability was evaluated by 3-[4,5-dimethylthiazol-2-yl]-2,5-diphenyl tetrazolium bromide (MTT) assay based on the reduction of MTT (Sigma-Aldrich) by functional mitochondria to formazan, as described previously [9].

Flow cytometry analysis of apoptosis

The cardiomyocytes were harvested, stained with annexin V/PI as previously described [10], and analyzed for apoptosis by flow cytometry (Becton-Dickinson, Franklin Lakes, NJ, USA) at an excitation wavelength of $488 \mathrm{~nm}$ and an emission wavelength of $615 \mathrm{~nm}$, according to the manufacturer's instructions.

Table 1. Primers used for quantitative real-time RT-PCR. * These are the primers used for quantitative real-time RT-PCR. RT-primer was used for reverse transcription to obtain a cDNA segment of miR-1

\begin{tabular}{lll}
\hline Type & Name & Sequence \\
\hline RT-primer & miR-1 & 5'-GGCTGCCGACCGTGTCGTGGAGTCGGCAA \\
& & TTGGTCGGCAGCCATACACAC-3' \\
PCR-primer & miR-1-F & 5'-CTGTCACTCGAGCTGCTGGAATG-3' \\
& miR-1-R & 5'-ACCGTGTCGTGGAGTCGGCAATT-3' \\
& HDAC4-F & 5'-ATCAGAGACCCAATGCCAAT-3' \\
& HDAC4-R & 5'-AGCAGGTTTGACGCCTACAG-3' \\
& $\beta$-actin-R & 5'-ATGGTGGGTATGGGTCAGAAGG-3' \\
& $\beta$-actin-F & 5'-TGGCTGGGGTGTTGAAGGTC-3' \\
\hline
\end{tabular}

Table 2. The sequences of miR-1 mimic and scrambled RNA. * These are the sequences of miR-1 mimic and scrambled RNA, used to upregulate cardiomyocyte miR-1

\begin{tabular}{ll}
\hline Name & Sequence \\
\hline miR-1 mimic & 5'-UGGAAUGAAAGAAGUGUUAUACACUUCUUUACAU \\
& UCCAUU-3' \\
scrambled RNA & 5'-AUACACACUACUC77UACAUACCA-3' \\
\hline
\end{tabular}

Table 3. The sequences of siHDAC4 and scrambled RNA. * These are the sequences of siHDAC4 and scrambled RNA to knockdown cardiomyocyte HDAC4 protein

\begin{tabular}{ll}
\hline Name & Sequence \\
\hline siHDAC4-rat-1313 (S1)-F & 5'-CCCACUUCCUCUCUAUACATT-3' \\
siHDAC4-rat-1313 (S1)-R & 5'-UGUAUAGAGAGGAAGUGGGTT-3' \\
siHDAC4-rat-2537 (S2)-F & 5'-GGUGGAUAGUGAUACCAUATT-3' \\
siHDAC4-rat-2537 (S2)-R & 5'-UAUGGUAUCACUAUCCACCTT-3' \\
siHDAC4-rat-3014 (S3)-F & 5'-GGUCAUGCCAAUCGCAAAUTT-3' \\
siHDAC4-rat-3014 (S3)-R & 5'-UAUGGUAUCACUAUCCACCTT-3' \\
scrambled RNA-F & 5'-UUCUCCGAACGUGUCACGUdTdT-3' \\
scrambled RNA-R & 5'-ACGUGACACGUUCGGAGAAdTdT-3' \\
\hline
\end{tabular}




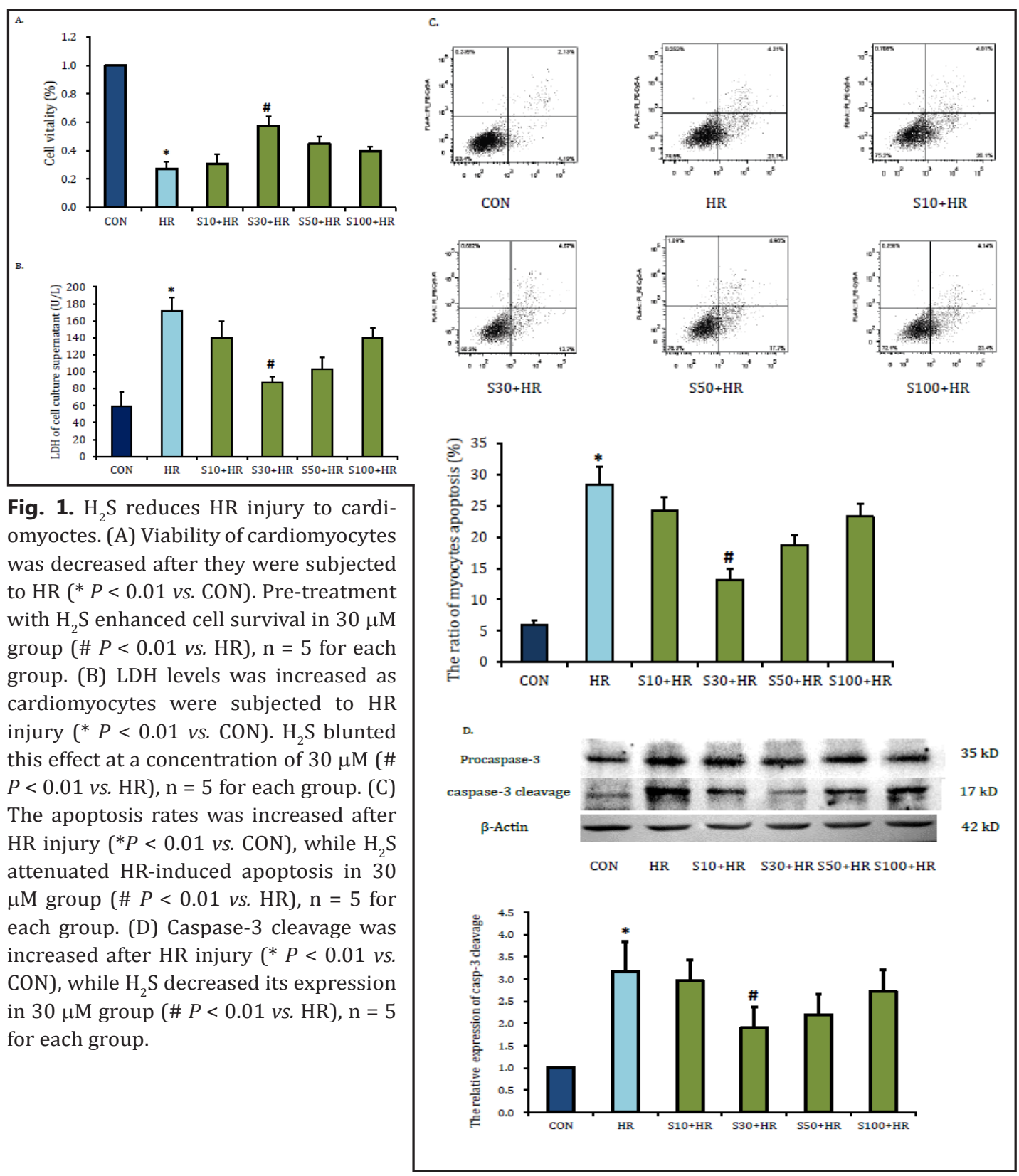

Infarct size measurement

The infarct size of the myocardium was measured as previously described [6]. The extent of myocardial infarction was evaluated after reperfusion. Representative photographs of midventricular cross sections of the Evans blue and TTC-stained hearts were taken. The infarct area was determined by computerized planimetry.

\section{TUNEL staining}

The myocardial tissue sample was sliced into 4- $\mu$ m-thick sections for apoptotic assessment with terminal deoxynucleotidyl transferase (TDT)-mediated DNA nick-end labeling (TUNEL) assay as previously described [11]. The apoptosis rate was calculated by randomly selecting five non-overlapping areas of the IR injury area at $400 \times$ high-power field. From these regions, the number of apoptotic cells and total cardiomyocytes were counted. The apoptosis rate refers to the number of apoptotic cells divided by the total number of cardiomyocytes $\times 100 \%$.

\section{KARGER}


Real-Time RT-PCR

Total RNA from cardiomyocytes was extracted and then reverse transcribed to generate cDNA. The mRNA of HDAC4 and miR-1 was quantified by quantitative real-time RT-PCR in duplicate samples using the Rotor Gene 3000 (Corbett Research, Sydney, Australia). The annealing temperature of miR-1 was $60^{\circ} \mathrm{C}$, and the annealing temperature of HDAC 4 and $\beta$-actin was $58^{\circ} \mathrm{C}$. The primer sequences are shown in Table 1.

\section{Western blotting analysis}

The protein expression of HDAC4, $\beta$-Actin, procaspase-3 and caspase-3 cleavage were detected by Western blotting analysis, as previously described $[12,13]$. All antibodies were purchased from Sigma (USA).

MiR-1 mimic synthesis and stimulation on cardiomyocytes

MiR-1 nucleotide (MIMAT0003125) and scrambled RNA were synthesized by Shanghai GenePharma Corporation (China) (Table 2). The dosages of mimic and scrambled RNA were determined according to the manual. The nucleotides were transfected with the siPORTNeoFX Transfection Agent (Invitrogen). MiR-1 was quantified in the different groups to determine the efficiency of transfection.

To see how miR-1 regulated HDAC4 expression, cardiomyocytes were divided into six groups: control group (CON group), mock transfected (MT, transfected with scrambled RNA) group and four $(10,30,50$ or $100 \mu \mathrm{M})$ mimic groups (transfected with miR-1 mimic). The RNA was transfected into the cells for $24 \mathrm{~h}$, and HDAC4 protein was measured by immunoblotting.

\section{RNA interference}

Using the bioinformatics method, three different loci small interfering RNA (siRNA) of HDAC4 (siHDAC4) were predicted and synthesized (Table 3). All the RNAs were custom-synthesized by Shanghai GenePharma Corporation. Transfection of siHDAC4 was performed by using siPORTNeoFx transfection agent according to the manual. The different siHDAC4 agents were transfected into the cardiomyocytes for $24 \mathrm{~h}$ respectively, and HDAC4 protein was measured by immunoblotting to confirm their interfering efficiency.

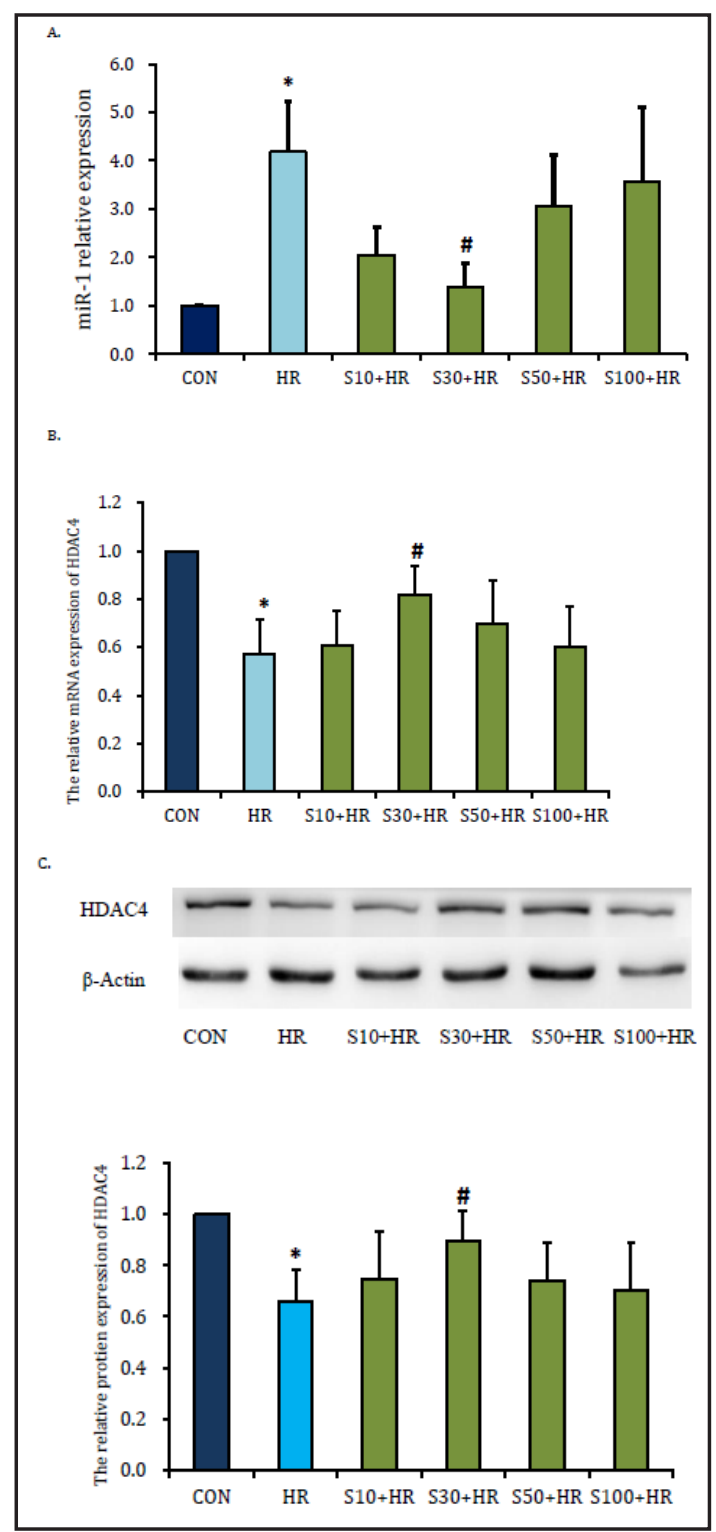

Fig. 2. The effects of $\mathrm{H}_{2} \mathrm{~S}$ on miR-1 and HDAC4 expressions in vitro. (A) MiR-1 expression was upregulated by $\mathrm{HR}(* P<0.001$ vs. $\mathrm{CON})$, and $\mathrm{H}_{2} \mathrm{~S}$ downregulated miR-1 expression in $30 \mu \mathrm{M}$ group ( $P<0.01 \mathrm{vs}$. HR), $n=5$ for each group. (B) Relative HDAC4 mRNA was downregulated by HR injury and upregulated by $\mathrm{H}_{2} \mathrm{~S}$ in $30 \mu \mathrm{M}\left({ }^{*} P<0.01\right.$ vs.CON; \# $P<0.05$ vs. HR), $\mathrm{n}=5$ for each group. (C) HDAC4 protein expression was downregulated by HR $(* P<0.01$ vs. CON) and upregulated by $\mathrm{H}_{2} \mathrm{~S}$ also in $30 \mu \mathrm{M}(\# P<0.05$ vs. HR), $\mathrm{n}=5$ for each group.

\section{Statistical analysis}

Quantitative data are presented as mean \pm standard error of the mean (SEM). Statistical significance was determined using one-way or two-way ANOVA. Significance was established at the $P<0.05$ level. 

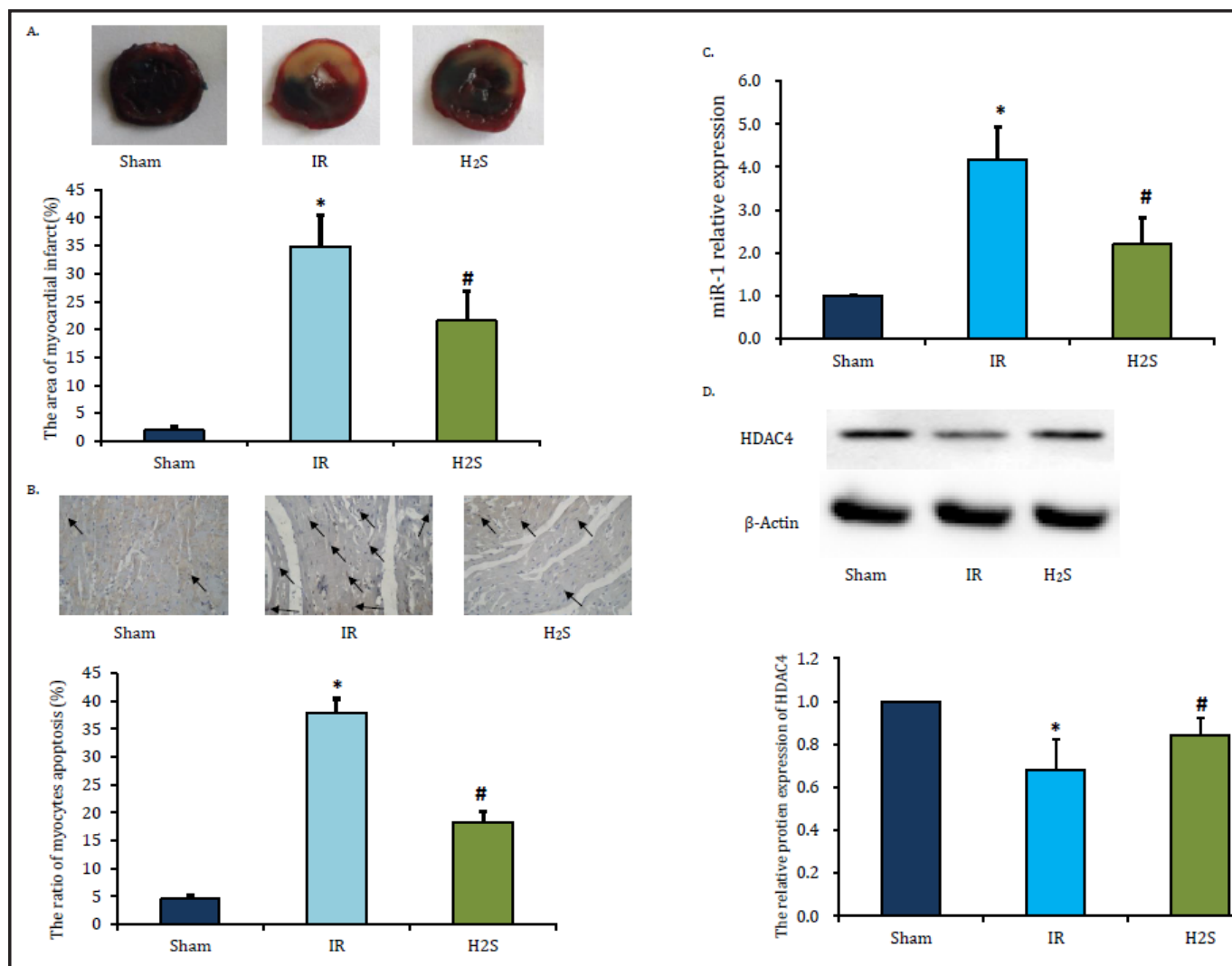

Fig. 3. The effects of $\mathrm{H}_{2} \mathrm{~S}$ on miR-1 and HDAC4 expressions in vivo. (A) Representative midmyocardial cross sections of TTC-stained hearts for IR. The dark blue area is the nonischemic zone; the white area is the infarcted tissue; the red area is the area at risk, ( ${ }^{*} P<0.001$ vs. Sham; \# $P<0.01$ vs. IR), $\mathrm{n}=5$ for each group. (B) In vivo, myocardial cell nuclei are stained blue; while the middle and late apoptotic cardiomyocytes nuclei are stained brown and defined as "TUNEL positive cells". TUNEL positive cells were increased in IR group ( ${ }^{*} P<0.001$ vs. Sham), and decreased after $\mathrm{H}_{2} \mathrm{~S}$ preconditioning (\# $P<0.01$

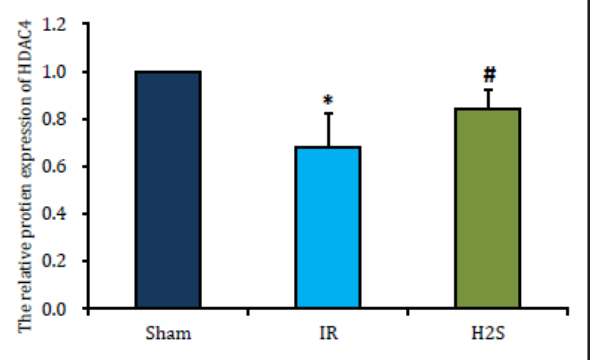
E.

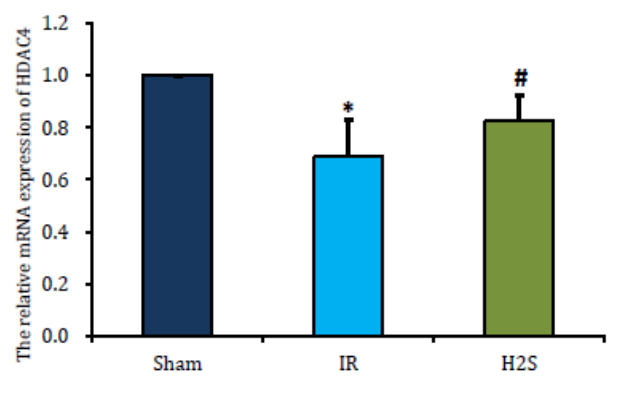

vs. IR), $\mathrm{n}=5$ for each group. (C) MiR-1 was up-regulated by HR ( ${ }^{*} P<0.001$ vs. Sham), and $\mathrm{H}_{2} \mathrm{~S}$ down-regulated it ( $P<<0.01$ vs. IR), $\mathrm{n}=5$ for each group. (D) Relative HDAC4 protein expression was down-regulated by IR ( ${ }^{*}<0.01$ vs. Sham) and was upregulated by $\mathrm{H}_{2} \mathrm{~S}$ ( $\# P<0.05$ vs. IR), $\mathrm{n}=5$ for each group. (E) Relative HDAC4 mRNA expression was down-regulated by IR injury ( $P<0.05 \mathrm{vs}$. Sham) and was upregulated by $\mathrm{H}_{2} \mathrm{~S}$ (\# $P<0.05$ vs. IR), $\mathrm{n}=5$ for each group.

\section{Results}

$\mathrm{H}_{2} \mathrm{~S}$ attenuates HR injury to cardiomyocytes

Cell viability was decreased and LDH was increased significantly after HR. Cell viability was increased by approximately $30.9 \%$ in the $30 \mu \mathrm{M}$ NaHS group compared with HR group (Fig. 1A). Meanwhile, LDH in $\mathrm{H}_{2} \mathrm{~S}$ groups was also decreased in the $30 \mu \mathrm{M}$ NaHS group (Fig. 1B). $\mathrm{H}_{2} \mathrm{~S}$ attenuated cardiomyocyte apoptosis in the $30 \mu \mathrm{M}$ NaHS group (Fig. 1C). Caspase- 3 cleavage was also decreased in $30 \mu \mathrm{M}$ group (Fig. 1D). These data confirmed that $30 \mu \mathrm{M} \mathrm{H} \mathrm{H}_{2} \mathrm{~S}$ could protect myocardial cells against HR injury by inhibiting cell apoptosis, so $30 \mu \mathrm{M}$ was chosen for the further experiments. 


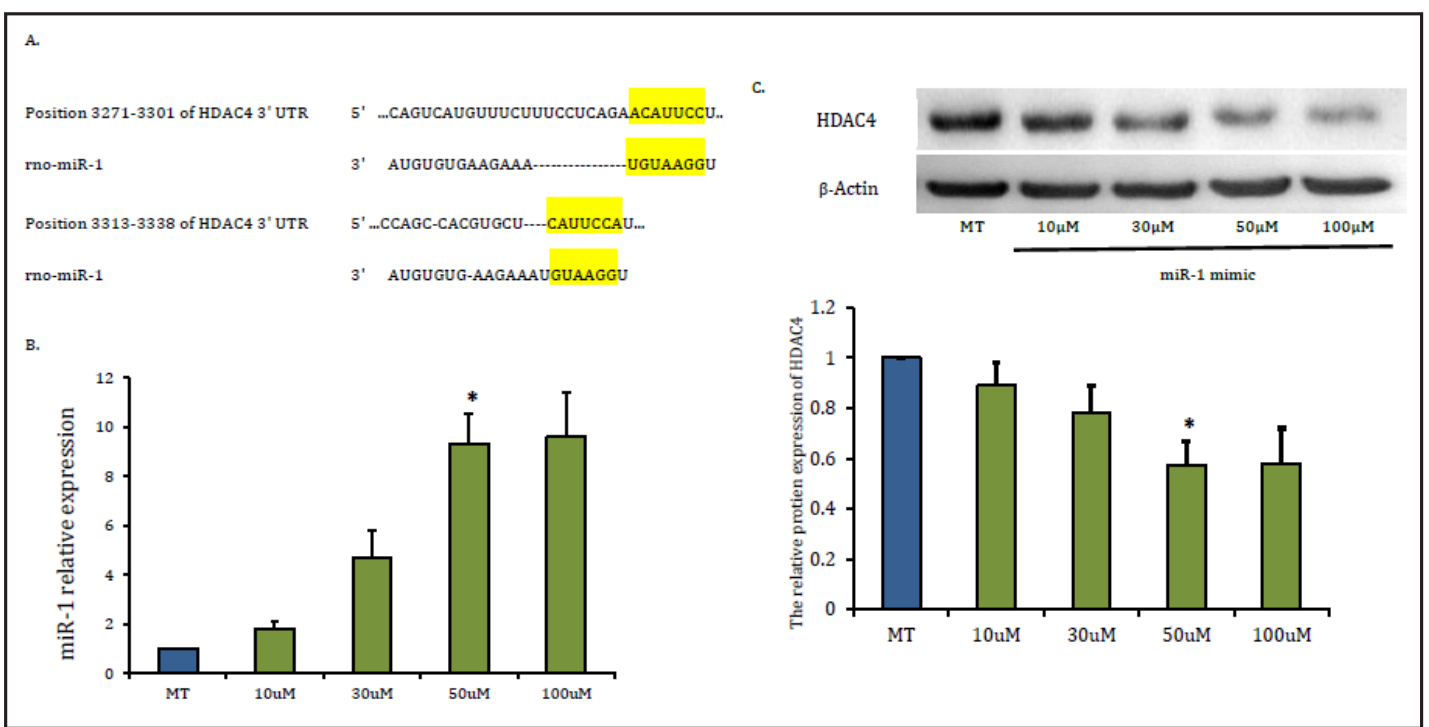

Fig. 4. MiR-1 mimic inhibits the expression of HDAC4. (A) The potential binding sites between miR-1 and the 3' UTR of HDAC4 mRNA. The complementary nucleotides between miR-1 and the target region of HDAC4 3'-UTR were highlighted in yellow fluorescence. (B) MiR-1 was upregulated by the miR-1 mimic at an effective dose of $50 \mu \mathrm{M}\left({ }^{*} P<0.001\right.$ vs. MT), $\mathrm{n}=5$ for each group. (C) MiR-1 downregulated HDAC4 protein expressions in a concentration-dependent manner with $50 \mu \mathrm{M}$ being the most effective $\left(^{*} P<0.01\right.$ vs. MT), $\mathrm{n}=5$ for each group.

\section{$\mathrm{H}_{2} \mathrm{~S}$ regulates $\mathrm{miR}$ - 1 and HDAC4 expression in vitro}

MiR-1 expression was upregulated after HR treatment. $30 \mu \mathrm{M}$ NaHS downregulated miR-1 expression significantly (Fig. 2A). Meanwhile, HDAC4 mRNA and protein expression was also downregulated after $\mathrm{HR}$, while both were preserved by $30 \mu \mathrm{M} \mathrm{H}_{2} \mathrm{~S}$ (Fig. 2B and C).

\section{$\mathrm{H}_{2} \mathrm{~S}$ regulates miR-1 and HDAC-4 expression in vivo}

Similar to the findings in vitro, the in vivo infarct size in $\mathrm{H}_{2} \mathrm{~S}$ group was smaller than that in IR group (Fig. 3A). The number of TUNEL staining positive cardiomyocytes in IR group was greater than that in Sham group, while $\mathrm{H}_{2} \mathrm{~S}$ significantly reduced the number of apoptotic cells compared with IR group (Fig. 3B). MiR-1 expression was up-regulated by IR and down-regulated by $\mathrm{H}_{2} \mathrm{~S}$ during IR in vivo (Fig. 3C). In contrast, HDAC4 mRNA and protein expressions were down-regulated by IR, while both were preserved by $\mathrm{H}_{2} \mathrm{~S}$ (Fig. 3D and E).

\section{MiR-1 regulates HDAC4 protein expression}

According to Targetscan and miR Base algorithms, two target sites at 3271-3301 and 3313-3338 of HDAC4 3' UTR were recognized by miR-1 (Fig. 4A), suggesting that HDAC4 may be one of the miR-1 potential downstream target genes. Different concentrations of miR-1 mimic were transfected into cardiomyocytes. MiR-1 expression was the highest in 50 $\mu \mathrm{M}$ and $100 \mu \mathrm{M}$ groups. As there was no statistically significant difference between the two doses, $50 \mu \mathrm{M}$ mimic was selected for further experiments (Fig. 4B).

HDAC4 was decreased in a dose dependent manner in miR-1 mimic groups compared with the MT group. HDAC4 protein decreased the most significantly in $50 \mu \mathrm{M}$ group compared with that in MT (Fig. 4C). Thus, $50 \mu \mathrm{M}$ mimic was used for further experiments. These results indicated that miR-1 inhibited the synthesis of HDAC4 protein, probably through a posttranscriptional mechanism.

\section{MiR-1 is involved in $\mathrm{H}_{2} \mathrm{~S}$ protecting cardiomyocytes against apoptosis}

To investigate whether miR-1 was involved in the protective effect of $\mathrm{H}_{2} \mathrm{~S}$ against HR-induced apoptosis, miR-1 levels were upregulated by mimic. The cardiomycytes were transfected with miR-1 mimic, preconditioned with $30 \mu \mathrm{M}$ NaHS, and then subjected to HR. No significant difference in cell viability was observed between MT group and $\mathrm{MT}+\mathrm{H}_{2} \mathrm{~S}$ group. 


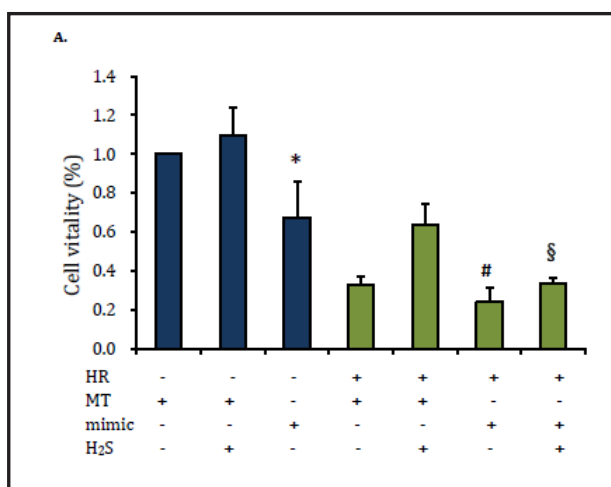

Fig. 5. MiR-1 abrogates the cardioprotective effect of $\mathrm{H}_{2} \mathrm{~S}$. (A) Cell viability in mimic group was decreased compared with MT group (* $P<0.05$ vs. MT). Cell viability in mimic + HR group was significantly decreased compared with MT+HR group ( $P<0.01$ vs. MT+HR). Cell viability in $\mathrm{HR}+$ mimic $+\mathrm{H}_{2} \mathrm{~S}$ group was decreased compared with $\mathrm{HR}+\mathrm{MT}+\mathrm{H}_{2} \mathrm{~S}$ group $\left(\S P<0.05 v s\right.$. $\mathrm{HR}+\mathrm{MT}+\mathrm{H}_{2} \mathrm{~S}$ ). As was the case with $\mathrm{HR}$ injury and mimic treatment, $\mathrm{H}_{2} \mathrm{~S}$ pretreatment did not upregulate myocardial cell viability significantly, $\mathrm{n}=5$ for each group. (B) Compared with MT group, cell apoptosis rate in mimic group was increased $(* P<0.05$ vs. MT). The cell apoptosis rate in mimic +HR group was increased compared with $\mathrm{MT}+\mathrm{HR}$ group (\# $P<0.01$ vs. MT+HR). The cell apoptosis rate in $\mathrm{HR}+$ mimic $+\mathrm{H}_{2} \mathrm{~S}$ group was increased compared with $\mathrm{HR}+\mathrm{MT}+\mathrm{H}_{2} \mathrm{~S}$ group $\left(\S P<0.05\right.$ vs. $\left.\mathrm{HR}+\mathrm{MT}+\mathrm{H}_{2} \mathrm{~S}\right)$. For $\mathrm{HR}$ injury and mimic treatment, $\mathrm{H}_{2} \mathrm{~S}$ pretreatment did not downregulate myocardial cell apoptosis significantly, $\mathrm{n}=5$ for each group. (C) MiR-1 mimic decreased the expression of HADC4 ( ${ }^{*} P<0.01$ vs. MT). After HR injury, HDAC4 in mimic+HR was lower than that in $\mathrm{MT}+\mathrm{H}_{2} \mathrm{~S}(\# P<0.05$ vs. MT+HR). HDAC4 in $\mathrm{HR}+$ mimic $+\mathrm{H}_{2} \mathrm{~S}$ group was decreased compared with $\mathrm{HR}+\mathrm{MT}+\mathrm{H}_{2} \mathrm{~S}$ group $(\S P<$ 0.05 vs. $\mathrm{HR}+\mathrm{MT}+\mathrm{H}_{2} \mathrm{~S}$ ). For HR injury and mimic treatment, $\mathrm{H}_{2} \mathrm{~S}$ pretreatment did not upregulate HDAC4 significantly, $\mathrm{n}=5$ for each group.

Compared with the MT group, cell viability in the mimic group was decreased, suggesting that upregulation of miR-1 was detrimental to cell function. Cell viability in mimic +HR group was decreased compared with that in MT+HR group. Cell viability in $\mathrm{HR}+$ mimic $+\mathrm{H}_{2} \mathrm{~S}$ group was decreased compared with that in $\mathrm{HR}+\mathrm{MT}+\mathrm{H}_{2} \mathrm{~S}$ group. After upregulation of miR-1 and $\mathrm{HR}$ injury, $\mathrm{H}_{2} \mathrm{~S}$ pretreatment did not upregulate cell viability significantly (Fig. 5A), suggesting that upregulating miR-1expression could partially abrogate the protective effect of $\mathrm{H}_{2} \mathrm{~S}$ on cardiomyocyte viability.

There was no significant difference in the apoptosis rate between MT group and MT $+\mathrm{H}_{2} \mathrm{~S}$ group. However, compared with MT group, the apoptosis rate in mimic group was increased, suggesting that upregulation of miR-1 could increase apoptosis. After HR, the apoptosis
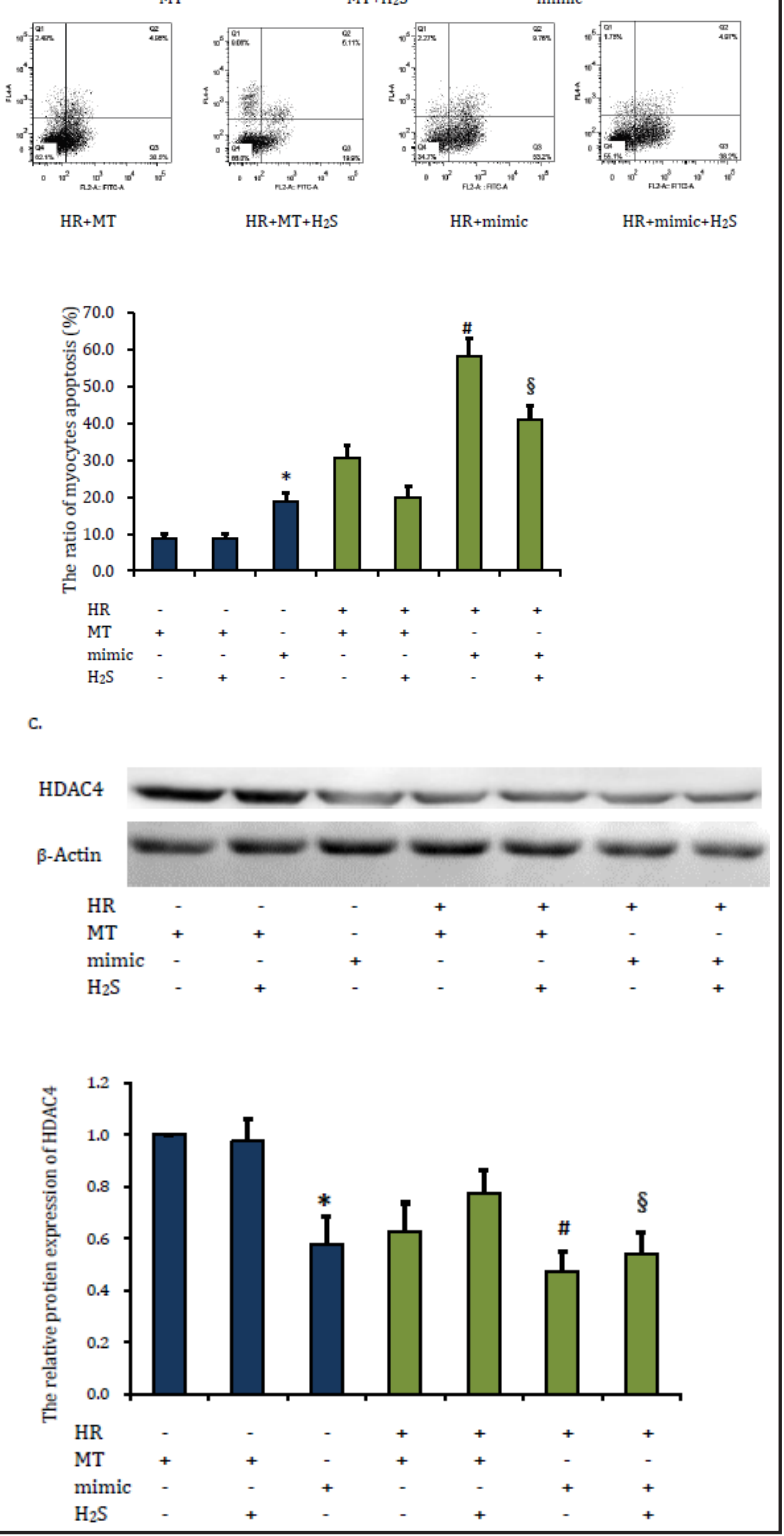


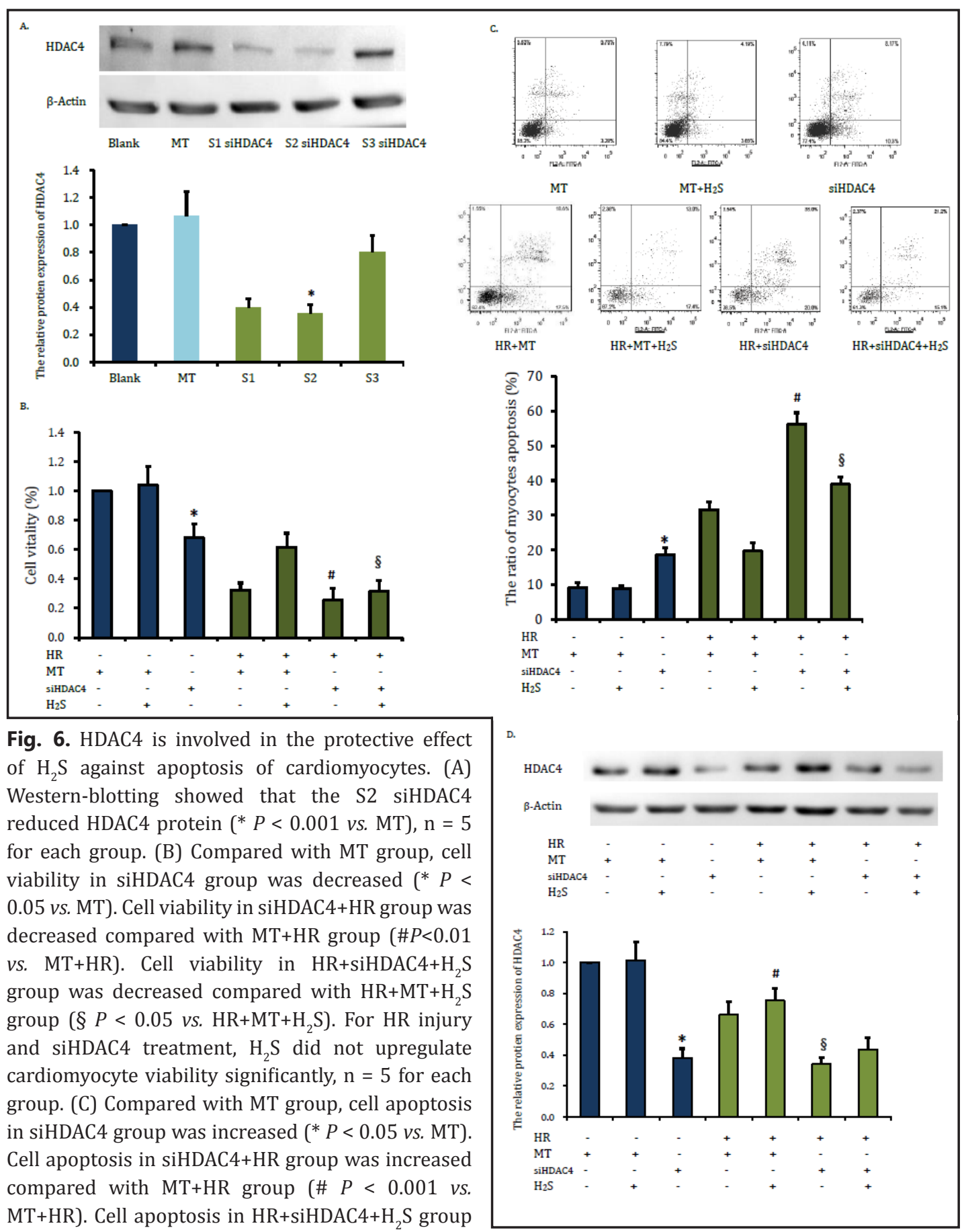
$\mathrm{MT}+\mathrm{HR})$. Cell apoptosis in $\mathrm{HR}+\mathrm{SiHDAC} 4+\mathrm{H}_{2} \mathrm{~S}$ group was increased compared with $\mathrm{HR}+\mathrm{MT}+\mathrm{H}_{2} \mathrm{~S}$ group $\left(\S P<0.01 v s . \mathrm{HR}+\mathrm{MT}+\mathrm{H}_{2} \mathrm{~S}\right)$. For HR and siHDAC4 treatment, $\mathrm{H}_{2} \mathrm{~S}$ pretreatment did not downregulate myocardial cell apoptosis significantly, $\mathrm{n}=5$ for each group. (D) HDAC4 siHDAC4 decreased the expression of HADC4 $\left({ }^{*} P<0.01 v s\right.$. MT). After HR, HDAC4 in siHDAC4+HR group was lower than that in $\mathrm{MT}+\mathrm{H}_{2} \mathrm{~S}$ group ( $\#<0.01$ vs. MT $+\mathrm{HR}$ ). HDAC4 in $\mathrm{HR}+\mathrm{siHDAC} 4+\mathrm{H}_{2} \mathrm{~S}$ group was significantly decreased compared with $\mathrm{HR}+\mathrm{MT}+\mathrm{H}_{2} \mathrm{~S}$ group $\left(\S P<0.05\right.$ vs. $\left.\mathrm{HR}+\mathrm{MT}+\mathrm{H}_{2} \mathrm{~S}\right)$. For HR injury and siHDAC4 treatment, $\mathrm{H}_{2} \mathrm{~S}$ pretreatment did not upregulate HDAC4 significantly, $\mathrm{n}=5$ for each group.

rate in mimic + HR group was significantly increased compared with that in MT+HR group. Similarly, after $\mathrm{H}_{2} \mathrm{~S}$ pretreatment and $\mathrm{HR}$, the apoptosis rate in $\mathrm{HR}+$ mimic $+\mathrm{H}_{2} \mathrm{~S}$ group was increased compared with that in $\mathrm{HR}+\mathrm{MT}+\mathrm{H}_{2} \mathrm{~S}$ group. After upregulation of miR-1 and HR 
injury, $\mathrm{H}_{2} \mathrm{~S}$ did not downregulate the cell apoptosis rate significantly. These results suggest that miR-1 could partially abrogate the protective effects of $\mathrm{H}_{2} \mathrm{~S}$ on cardiomyocyte apoptosis (Fig. 5B).

MiR-1 mimic decreased HADC4 expression. When cardiomyocytes were subjected to $\mathrm{HR}$ in combination with miR-1 overexpression, $\mathrm{H}_{2} \mathrm{~S}$ pretreatment could not normalize the expression level of HDAC4 (Fig. 5C).

HDAC4 is involved in $\mathrm{H}_{2} \mathrm{~S}$ protecting cardiomyocytes against apoptosis

To investigate whether HDAC4 was involved in the protective effect of $\mathrm{H}_{2} \mathrm{~S}$ against HRinduced apoptosis, HDAC4 levels were downregulated by siHDAC4. HDAC4 protein was reduced significantly in S1 siHDAC4 and S2 siHDAC4 groups, especially in S2 siHDAC4 group, and therefore S2 siHDAC4 was selected for subsequent experiments (Fig. 6A).

The cardiomycytes were transfected with siHDAC4, preconditioned with $30 \mu \mathrm{M}$ NaHS, and then subjected to HR. No significant difference in cell viability was found between MT group and $\mathrm{MT}+\mathrm{H}_{2} \mathrm{~S}$ group. Compared with MT group, cell viability in siHDAC4 group was decreased, suggesting that downregulation of HDAC4 could reduce cell vitality. In addition, cell viability in siHDAC4+HR group was decreased compared with that in MT+HR group. Following $\mathrm{H}_{2} \mathrm{~S}$ pretreatment and HR, cell viability in $\mathrm{HR}+\mathrm{SiHDAC} 4+\mathrm{H}_{2} \mathrm{~S}$ group was decreased compared with that in $\mathrm{HR}+\mathrm{MT}+\mathrm{H}_{2} \mathrm{~S}$ group. Moreover, following HR injury and siHDAC4 transfection, $\mathrm{H}_{2} \mathrm{~S}$ pretreatment did not upregulate myocardial cell viability significantly (Fig. 6B).

No significant difference in cell apoptosis was found between MT group and MT+ $+\mathrm{H}_{2} \mathrm{~S}$ group. Compared with MT group, cell apoptosis in siHDAC4 group was increased, suggesting that downregulation of HDAC4 could increase cell apoptosis. Following HR damage, cell apoptosis in siHDAC4+HR group was increased compared with MT+HR group. Cell apoptosis in $\mathrm{HR}+\operatorname{siHDAC} 4+\mathrm{H}_{2} \mathrm{~S}$ group was increased compared with that in $\mathrm{HR}+\mathrm{MT}+\mathrm{H}_{2} \mathrm{~S}$ group. Following the same HR injury and siHDAC4 transfection, $\mathrm{H}_{2} \mathrm{~S}$ pretreatment did not upregulate cardiomyocytes apoptosis significantly (Fig. 6C).

siHDAC4 decreased the expression of HDAC4 in cardiomyocytes. Following siHDAC4 transfection and $\mathrm{HR}, \mathrm{H}_{2} \mathrm{~S}$ did not normalize HDAC4 expression significantly (Fig. 6D).

\section{Discussion}

Blood perfusion to the ischemic myocardium is known to increase cardiac structural damage and functional impairment, which is known as IR injury [12,14]. In recent years, studies have shown that some drugs could protect the heart from IR Injury by attenuating mycardiocytes apoptosis $[15,16]$. It also has been found that $\mathrm{H}_{2} \mathrm{~S}$ has an important endogenous protective effect against myocardial IR injury, thus improving the heart function by reducing the infarct size and inhibiting apoptosis of myocardial cells [17] $\mathrm{H}_{2} \mathrm{~S}$ is a gasotransmitter, and the third endogenous gaseous molecule that has a biological activity after exposure to nitric oxide (NO) and carbon monoxide (CO) [18], followed by ammonium which has been suggested as the fourth one [19].

$\mathrm{H}_{2} \mathrm{~S}$ is widely and extensively involved in many important physiolopathological processes in multiple organs [20]. The protective effect of $\mathrm{H}_{2} \mathrm{~S}$ on myocardial IR injury might be closely related to intervening the expression of apoptosis-related genes [21]. Some $\mathrm{H}_{2} \mathrm{~S}$ related drugs have been entered into Phase II clinical trials and are expected to be put into clinical use in the near future.

In this study, the $\beta$-actin gene was chosen as the housekeeping gene. Other researchers used $\beta$-actin as a housekeeping gene in neonatal rat cardiomyocytes [13]. In our laboratory there was some experience in terms of detection of $\beta$-actin in neonatal cardiomyocytes [12]. It was found that $30 \mu \mathrm{M} \mathrm{H}_{2} \mathrm{~S}$ exhibited an obvious protective effect. Nevertheless, our results demonstrated that $30 \mu \mathrm{M} \mathrm{H}_{2} \mathrm{~S}$ could inhibit myocardial apoptosis, reduce the myocardial IR injury and promote cardiac function.

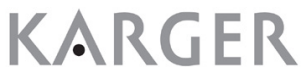


MiRs are known to play an important role in the regulation of gene expression at post transcriptional levels. MiR-21 could regulate endogenous $\mathrm{H}_{2} \mathrm{~S}$ by repressing the cystathionine- $\gamma$-lyase expression [22]. However whether exogenous $\mathrm{H}_{2} \mathrm{~S}$ could regulate cell pathophysiology by miRs remains uncertain. In our previous study, we found that miR-1 expression underwent a significant change in IR group compared with $\mathrm{H}_{2} \mathrm{~S}$ preconditioned group.

Histone deacetylation alters chromosome structure and affects transcription factor access to DNA. This protein does not bind to DNA directly but through transcription factors, MEF2C and MEF2D, which play a critical role in transcriptional regulation [23]. HDAC4 is known to play a clear anti-apoptotic role in various tumor cells. HDAC4 regulates neuronal survival in normal and diseased retinas [24]. However, the role of HDAC4 in cardiomyocytes remains unclear. Our study provided direct evidence to show the involvement of HDAC4 in apoptosis of cardiomyocytes.

Our results showed that miR-1 expression was decreased in cardiomyocytes pretreated with $30 \mu \mathrm{M} \mathrm{H}_{2} \mathrm{~S}$. In contrast, $30 \mu \mathrm{M} \mathrm{H}_{2} \mathrm{~S}$ could upregulate the expression of HDAC4, which is consistent with the protective effect of $\mathrm{H}_{2} \mathrm{~S}$ and inhibition of myocardial IR-induced apoptosis of cardiomyoctyes. Together with the prediction results of bioinformatics, these data suggest that miR-1 might regulate the expression of HDAC4. In order to confirm this hypothesis, cardiomyocytes were transfected with miR-1 mimic. It was found that the protein expression of HDAC4 correlated with the amount of the transfected mimic, indicating that the miR-1 could regulate HDAC4 protein expression, which suggests that HDAC4 is one of the downstream target genes of miR-1. The generation process of miRNA is complex, and $\mathrm{H}_{2} \mathrm{~S}$ may affect the key enzymes of formation and maturation to regulate the miR-1 expression, such as Drosha and Dicer. Its exact mechanism is unclear and will be explored in our ongoing study.

To study whether the miR-1-HDAC4 signal pathway was involved in the protective effect of $\mathrm{H}_{2} \mathrm{~S}$ against myocardial IR injury, myocardial cells were transfected with miR-1 mimic or siHDAC4. It was found that miR-1 mimic could bind to the corresponding specific site of the target genes, and played a regulatory role in the post-transcriptional level as the endogenous miR-1. The protective and anti-apoptotic effects of $\mathrm{H}_{2} \mathrm{~S}$ were significantly reduced in these mimic transfected cells' suggesting that miR-1 was involved in the protective effect of $\mathrm{H}_{2} \mathrm{~S}$. Similarly, in these cells transfected by siHDAC4, the protective and anti-apoptotic effects of $\mathrm{H}_{2} \mathrm{~S}$ were also significantly reduced, suggesting that HDAC4 was also involved in the protective effect of $\mathrm{H}_{2} \mathrm{~S}$.

In summary, our results indicate that miR-1-HDAC4 signaling pathway is involved in the inhibitory effect of $\mathrm{H}_{2} \mathrm{~S}$ against IR-induced apoptosis of cardiomyocytes.

\section{Funding sources}

This work was supported by the National Nature Science Foundation of China (81600194, 81200181and 81270419), Nature Science Foundation of Science and Technology Commission of Shanghai municipality (15411960100), the Key Project of Chinese People's Liberation Amy (BWS13J012, AWS15J002), the Hospital development center of Shenkang (SHDC12014107), the Shanghai Municipal Commission of Health and Family Planning (2012Y137) , Scholarship of Earl Bakken (EEBK01) and Translational medicine fund of Changzheng hospital (2015CZZH08).

\section{Disclosure Statement}

The authors have no conflicts of interest to disclose. 


\section{Cellular Physiology Cell Physiol Biochem 2017;41:10-21

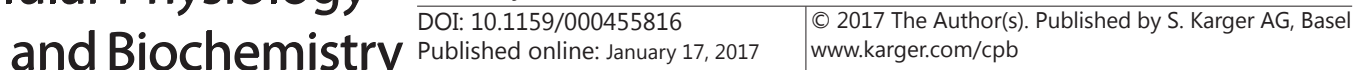

Kang et al.: $\mathrm{H}_{2} \mathrm{~S}$ Protects Cardiomyocytes through MiR-1-Regulated HDAC4

\section{References}

1 Xiao J, Zhu X, Kang B, Xu J, Wu L, Hong J, Zhang Y, Ni X, Wang Z: Hydrogen Sulfide Attenuates Myocardial Hypoxia-Reoxygenation Injury by Inhibiting Autophagy via mTOR Activation. Cell Physiol Biochem 2015;37:2444-2453.

2 Oliveira-Carvalho V, da Silva MM, Guimaraes GV, Bacal F, Bocchi EA: MicroRNAs: new players in heart failure. Mol Biol Rep 2013;40:2663-2670.

-3 Duan L, Xiong X, Liu Y, Wang J: miRNA-1: functional roles and dysregulation in heart disease. Mol Biosyst 2014;10:2775-2782.

4 Kang B, Hong J, Xiao J, Zhu X, Ni X, Zhang Y, He B, Wang Z: Involvement of miR-1 in the protective effect of hydrogen sulfide against cardiomyocyte apoptosis induced by ischemia/reperfusion. Mol Biol Rep 2014;41:6845-6853.

5 Winbanks CE, Beyer C, Hagg A, Qian H, Sepulveda PV, Gregorevic P: miR-206 represses hypertrophy of myogenic cells but not muscle fibers via inhibition of HDAC4. PLoS One 2013;8:e73589.

6 Jian X, Xiao-yan Z, Bin H, Yu-feng Z, Bo K, Zhi-nong W, Xin N: MiR-204 regulate cardiomyocyte autophagy induced by hypoxia-reoxygenation through LC3-II. Int J Cardiol 2011;148:110-112.

-7 Yao LL, Huang XW, Wang YG, Cao YX, Zhang CC, Zhu YC: Hydrogen sulfide protects cardiomyocytes from hypoxia/reoxygenation-induced apoptosis by preventing GSK-3beta-dependent opening of mPTP. Am J Physiol Heart Circ Physiol 2010;298:H1310-1319.

-8 Prentice HM, Moench IA, Rickaway ZT, Dougherty CJ, Webster KA, Weissbach H: MsrA protects cardiac myocytes against hypoxia/reoxygenation induced cell death. Biochem Biophys Res Commun 2008;366:775-778.

9 Kishi T, Cloutier SM, Kundig C, Deperthes D, Diamandis EP: Activation and enzymatic characterization of recombinant human kallikrein 8. Biol Chem 2006;387:723-731.

10 Chen CJ, Yu W, Fu YC, Wang X, Li JL, Wang W: Resveratrol protects cardiomyocytes from hypoxia-induced apoptosis through the SIRT1-Fox01 pathway. Biochem Biophys Res Commun 2009;378:389-393.

11 Xin W, Lu X, Li X, Niu K, Cai J: Attenuation of endoplasmic reticulum stress-related myocardial apoptosis by SERCA2a gene delivery in ischemic heart disease. Mol Med 2011;17:201-210.

12 Cong B, Wang L, Zhu X, Li X, Liu B, Ni X: SGK1 is involved in cardioprotection of urocortin-1 against hypoxia/reoxygenation in cardiomyocytes. Can J Cardiol 2014;30:687-695.

13 Otani H, Yoshioka K, Nishikawa H, Inagaki C, Nakamura T: Involvement of protein kinase C and RhoA in protease-activated receptor 1-mediated F-actin reorganization and cell growth in rat cardiomyocytes. J Pharmacol Sci 2011;115:135-143.

14 Yang J, Guo X, Ding JW, Li S, Yang R, Fan ZX, Yang CJ: RP105 Protects Against Apoptosis in Ischemia/ Reperfusion-Induced Myocardial Damage in Rats by Suppressing TLR4-Mediated Signaling Pathways. Cell Physiol Biochem 2015;36:2137-2148.

15 Wu H, Ye M, Yang J, Ding J, Dong W, Wang X: Nicorandil Protects the Heart from Ischemia/Reperfusion Injury by Attenuating Endoplasmic Reticulum Response-induced Apoptosis Through PI3K/Akt Signaling Pathway. Cell Physiol Biochem 2015;35:2320-2332.

-16 Wang X, Cao Y, Shen M, Wang B, Zhang W, Liu Y, He X, Wang L, Xia Y, Ding M, Xu X, Ren J: DIDS reduces ischemia/reperfusion-induced myocardial injury in rats. Cell Physiol Biochem 2015;35:676-688.

17 Zhang Y, Li H, Zhao G, Sun A, Zong NC, Li Z, Zhu H, Zou Y, Yang X, Ge J: Hydrogen sulfide attenuates the recruitment of $\mathrm{CD} 11 \mathrm{~b}(+) \mathrm{Gr}-1(+)$ myeloid cells and regulates Bax/Bcl-2 signaling in myocardial ischemia injury. Sci Rep 2014;4:4774.

18 Wang R: Two's company, three's a crowd: can H2S be the third endogenous gaseous transmitter? FASEB J 2002;16:1792-1798.

19 Wang R: Gasotransmitters: growing pains and joys. Trends Biochem Sci 2014;39:227-232.

20 Wang R: Physiological implications of hydrogen sulfide: a whiff exploration that blossomed. Physiol Rev 2012;92:791-896.

21 Hu Y, Chen X, Pan TT, Neo KL, Lee SW, Khin ES, Moore PK, Bian JS: Cardioprotection induced by hydrogen sulfide preconditioning involves activation of ERK and PI3K/Akt pathways. Pflugers Arch 2008;455:607616.

-22 Yang G, Pei Y, Cao Q, Wang R: MicroRNA-21 represses human cystathionine gamma-lyase expression by targeting at specificity protein-1 in smooth muscle cells. J Cell Physiol 2012;227:3192-3200.

23 Bottomley MJ, Lo Surdo P, Di Giovine P, Cirillo A, Scarpelli R, Ferrigno F, Jones P, Neddermann P, De Francesco R, Steinkuhler C, Gallinari P, Carfi A: Structural and functional analysis of the human HDAC4 catalytic domain reveals a regulatory structural zinc-binding domain. J Biol Chem 2008;283:26694-26704.

24 Chen B, Cepko CL: HDAC4 regulates neuronal survival in normal and diseased retinas. Science 2009;323:256-259. 